\title{
A Low Critical Event Rate Despite a High Abnormal Event Rate in Patients with Cardiac Implantable Electric Devices Followed Up by Remote Monitoring
}

\author{
Yoshimasa Morimoto ${ }^{1}$, Nobuhiro Nishii ${ }^{2}$, Saori Tsukuda ${ }^{1}$, Satoshi Kawada ${ }^{1}$, \\ Masakazu Miyamoto ${ }^{1}$, Akihito Miyoshi ${ }^{1}$, Koji Nakagawa ${ }^{1}$, Atsuyuki Watanabe ${ }^{1}$, \\ Kazufumi Nakamura ${ }^{1}$, Hiroshi Morita ${ }^{2}$ and Hiroshi Ito ${ }^{1}$
}

\begin{abstract}
:
Objective Remote monitoring (RM) of cardiac implantable electric devices (CIEDs) has been advocated as a healthcare standard. However, expert consensus statements suggest that all patients require annual face-toface follow-up consultations at outpatient clinics even if RM reveals no episodes. The objective of this study was to determine the critical event rate after CIED implantation through RM.

Methods This multicenter, retrospective, cohort study evaluated patients with pacemakers (PMs), implantable cardioverter defibrillators (ICDs), or cardiac resynchronization therapy defibrillator (CRT-Ds) and analyzed whether or not the data drawn from RM included abnormal or critical events.

Patients A total of 1,849 CIED patients in 12 hospitals who were followed up by the RM center in Okayama University Hospital were included in this study.

Results During the mean follow-up period of 774.9 days, 16,560 transmissions were analyzed, of which $11,040(66.7 \%)$ were abnormal events and only $676(4.1 \%)$ were critical events. The critical event rate in the PM group was significantly lower than that in the ICD or CRT-D groups $(0.9 \%$ vs. $5.0 \%$ or $5.9 \%, \mathrm{p}<0.001)$. A multivariate analysis revealed that ICD, CRT-D, and a low ejection fraction were independently associated with critical events. In patients with ICD, the independent risk factors for a critical event were old age, low ejection fraction, Brugada syndrome, dilated phase hypertrophic cardiomyopathy and arrhythmogenic right ventricular cardiomyopathy.

Conclusion Although abnormal events were observed in two-thirds of the transmitted RM data, the critical event rate was $<1 \%$ in patients with a PM, which was lower in comparison to the rates in patients with ICDs or CRT-Ds. A low ejection fraction was an independent predictor of critical events.
\end{abstract}

Key words: remote monitoring, pacemaker, implantable cardioverter defibrillator, cardiac resynchronization therapy with defibrillator

(Intern Med 58: 2333-2340, 2019)

(DOI: 10.2169/internalmedicine.1905-18)

\section{Introduction}

Remote monitoring (RM) of cardiac implantable electric devices (CIEDs) has been rapidly advocated as a new healthcare standard for patients with CIEDs. Several large, prospective, randomized trials of RM of patients with CIEDs have demonstrated its safety, feasibility, efficacy, as well as an association with improved survival (1-4). Expert consensus statements $(5,6)$ suggest that all patients with CIEDs should be offered RM as part of the standard followup management strategy. However, expert consensus state-

${ }^{1}$ Department of Cardiovascular Medicine, Okayama University Graduate School of Medicine, Dentistry, and Pharmaceutical Sciences, Japan and ${ }^{2}$ Department of Cardiovascular Therapeutics, Okayama University Graduate School of Medicine, Dentistry, and Pharmaceutical Sciences, Japan Received: August 1, 2018; Accepted: February 21, 2019; Advance Publication by J-STAGE: May 22, 2019 Correspondence to Dr. Nobuhiro Nishii,nnishi@md.okayama-u.ac.jp and nnnnishii2001@yahoo.co.jp 
ments $(5,6)$ also suggest that all patients require annual face-to-face follow-up examinations at outpatient clinics even if RM reveals no episodes. Because the number of CIEDs has been constantly increasing $(7,8)$, the workload of outpatient clinics has not been markedly reduced. As previously reported, various abnormal events are detected by CIEDs follow-up, including atrial tachyarrhythmia (ATA), ventricular tachyarrhythmia (VTA), lead failure, and abnormal battery events (1-4). However, the number of CIED patients who did not require intervention and did not need to visit an outpatient clinic was very large. If the selection of patients for CIED follow-up could be limited to those who require intervention, such as those who require additional medication or a change of medication, a change in programming, implantation of new leads, or the exchange of generator, the workload involved would dramatically decrease. To do this, determining the critical event rate in various categories and the categories that should be the focus of attention is important. The aim of the study was to evaluate whether the critical event rate differed according to various factors, including age, sex, CIED type and manufacturer, underlying heart disease, and ejection fraction.

\section{Materials and Methods}

\section{Patient population}

CIED patients in 12 hospitals (The Sakakibara Heart Institute of Okayama, Okayama Medical Center, Fukuyama Cardiovascular Hospital, Fukuyama City Hospital, Iwakuni Medical Center, Tsuyama Chuo Hospital, Kagawa Prefectural Central Hospital, Kochi Medical Center, Takamatsu Red Cross Hospital, Okayama Red Cross Hospital, Onomichi City Hospital, and Okayama University Hospital) who were followed up by the RM center in Okayama University Hospital were included in this study. The CIEDs included pacemakers (PM), implantable cardioverter defibrillators (ICDs), and cardiac resynchronization therapy defibrillators (CRT-D). The RM systems were based on periodic remote follow-ups with automatic alerts [Medtronic CareLink (MCL), Minneapolis, USA; Abbott Medical Merlin (AMM) (9), Sylmar, USA; Boston Scientific Latitude (BSL), St. Paul, USA; BIOTRONIK Home Monitoring (BHM), Berlin, Germany]. Patients who could not visit any hospital were excluded. All patients gave their written informed consent for RM, and the study protocol was approved by the Institutional Review Board and/or the Medical Ethics Committee of each hospital.

\section{Study design and event definitions}

This was a retrospective, multicenter, cohort study. RM data were manually or automatically transmitted based on each RM system. The durations of the periodic transmission schedules were different (every 1-4 months) in each hospital. All data transmitted by RM were analyzed and summarized by medical engineers and doctors in the RM center, focusing mainly on arrhythmic and device-related events. Abnormal events were defined as ATA events, VTA events, abnormal battery voltage events (reaching less than 1 year of expected battery life or $<10 \%$ ), electrical abnormal events related to the leads, and others, based on the diagnosis of each device. Although the CIED diagnosis of tachyarrhythmia was sometimes wrong, the CIED diagnosis was unchanged. The reasons were that it was difficult to precisely diagnose what the tachycardia was, even though it was carefully analyzed, and the focus of this study was on critical events, not abnormal events. Only the numbers of ATA, VTA, and abnormal battery events are shown because there were many manufacturer-specific abnormal events, including a short-VV-interval counter, noise reversion, OptiVol, CorVue, and the count of premature ventricular contractions.

Abnormal events were also classified as critical or noncritical events. Critical events were defined as appropriate/ inappropriate ICD therapy (including ICD shock or antitachycardia pacing), battery depletion reaching the elective replacement indicator, lead impedance abnormality (increase in pace/sense conductor impedance $>1,500 \Omega$ or decrease in pace/sense conductor impedance $<200 \Omega$, change in highvoltage lead impedance $>125$ or $<20 \Omega$ ), non-physiological noise, atrial or ventricular sensing failure, atrial or ventricular pacing failure, reduction of $\mathrm{P}$ - or R-wave sensing amplitude by $50 \%$ of the safety margin, acutely increased atrial or ventricular pacing threshold (capture threshold $>5 \mathrm{~V}$ or an increase in capture threshold $>2 \mathrm{~V}$ from baseline $<1 \mathrm{~V}$ ), sustained ventricular tachycardia out of the therapy zone, and others. Previous randomized studies reported that CIEDs could detect subclinical atrial fibrillation (AF) (10) much earlier $(1,3,6,11)$. However, because the strategy for antithrombotic therapy of subclinical AF has not been established, these events were not included as critical events in the present study. Similarly, alerts related to heart failure were excluded. Previous trials $(12,13)$ demonstrated the efficacy of RM as a diagnostic tool for heart failure. However, controversial results $(14,15)$ have also been reported, and the clinical diagnostic parameters have not been well established. The expert consensus statement (6) indicates that the use of RM as a diagnostic tool for heart failure is a class IIb recommendation.

\section{Statistical analysis}

Continuous data were summarized as the mean and standard deviation, were compared among groups using the Kruskal-Wallis test. Categorical data were summarized as the frequencies and percentages, and were compared using Pearson's chi-squared test. To account for correlations between repeated transmission measurements within patients, the generalized estimating equation (GEE) was used with a compound symmetry working correlation structure. Transmission data were compared among groups, and $\mathrm{p}$ values were calculated using GEE models. Multivariate analyses were performed to evaluate the correlations between covariates and critical events, and odds ratios with their 95\% con- 


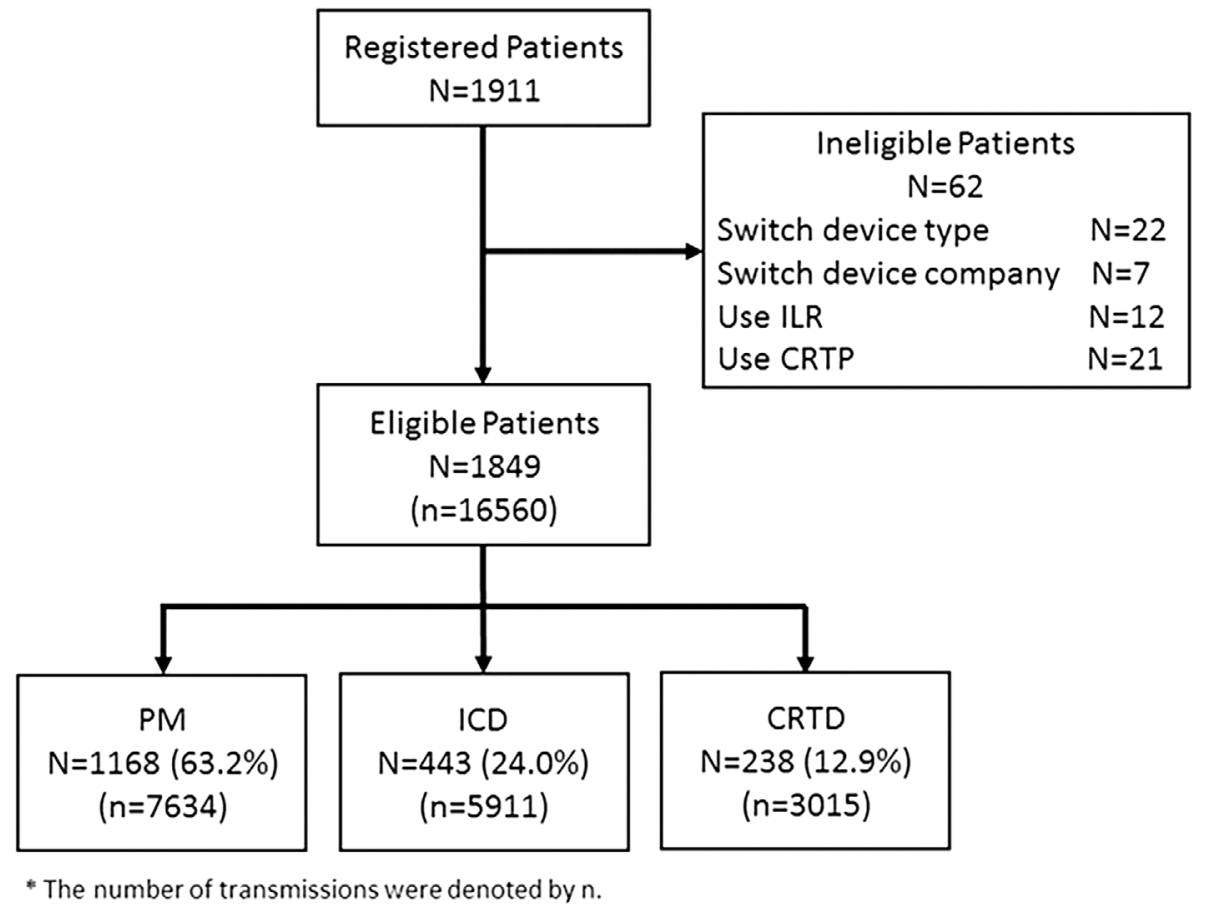

Figure. Patient population. Twelve patients with implantable loop recorders, 22 patients whose CIED generator type was exchanged, 7 patients whose generator manufacturer was changed, and 21 patients with CRT-P were excluded. The remaining 1,849 patients, including 1,168 PM, 443 ICD, and 238 CRT-D patients, were enrolled in this study. CIED: cardiac implantable electronic devices, CRTP: cardiac resynchronization therapy pacemaker, PM: pacemaker, ICD: implantable cardioverter defibrillator, CRT-D: cardiac resynchronization therapy defibrillator

fidence intervals and $\mathrm{p}$ values were calculated using a multivariate GEE model. All tests were two-sided, and $\mathrm{p}$ values of $<0.05$ were considered to indicate statistical significance. All statistical analyses were performed by SAS version 9.4 (SAS Institute, Cary, USA).

\section{Results}

\section{Patient population}

A total of 1,911 patients with CIEDs were registered in our database between August 2009 and March 2016. Fortyone patients were excluded due to loop recorder implantation $(n=12)$, change in CIED generator $(n=22)$, and change in generator manufacturer $(n=7)$. Twenty-one patients with cardiac resynchronization therapy pacemakers (CRT-Ps) were further excluded because the number of patients with CRT-Ps was too small to be analyzed accurately. Thus, 1,849 patients [PM, $\mathrm{n}=1,168$ (63.2\%); ICD, $\mathrm{n}=443$ (24.0\%); and CRT-D, n=238; (12.9\%)] patients, were analyzed (Figure).

\section{Patient characteristics and remote transmissions}

During a mean follow-up period of $774.9 \pm 652.9$ days, 16,560 transmissions in 1,849 patients were analyzed in the RM center. The mean age of the patients was $71.6 \pm 14.6$ years; the mean ejection fraction was $57.7 \pm 16.2 \%$, and $1,024(55.4 \%)$ patients were male. The baseline patient char- acteristics are shown in Table 1. Younger patients were more common in the ICD group than in the other groups, and fewer male patients were included in the PM group than in the other groups. The number of transmissions per patient was greater in ICD or CRT-D patients than in PM patients. The follow-up period in the PM group was shorter than that in the other groups. The proportions of CIED types differed among the manufacturers (Table 1).

\section{Abnormal events}

At least one abnormal event was observed in approximately two-thirds of the data transmissions $(11,040 / 16,565$, $66.7 \%$ ). The abnormal event rate in the CRT-D group was significantly greater than that in the other groups. Among the abnormal events, ATA events were most frequently observed $(n=5,981,36.1 \%)$, followed by VTA events $(n=3,753$, $22.7 \%$ ) and abnormal battery voltage events ( $n=334,2.0 \%)$. The ATA event rate was significantly lower in ICD patients, the VTA event rate was significantly lower in PM patients, and the abnormal battery voltage event rate was significantly lower in PM patients (Table 2).

\section{Critical events}

The critical event rate was only $4.1 \%(676 / 16,560)$. The critical event rate was significantly lower in PM patients $(0.9 \%)$ than in ICD $(6.6 \%)$ or CRT-D (7.3\%) patients. Among the three types of CIEDs, a major difference was observed in the critical event rate in ICD therapy (ICD, 
Table 1. Patient Characteristics.

\begin{tabular}{|c|c|c|c|c|c|}
\hline Variable & Overall $(\mathrm{n}=1,849)$ & $\mathrm{PM}(\mathrm{n}=1,168)$ & $\operatorname{ICD}(n=443)$ & CRT-D (n=238) & $\mathrm{p}$ value \\
\hline \multicolumn{6}{|l|}{ Gender } \\
\hline Male & $1,024(55.4)$ & $530(45.4)$ & $333(75.2)$ & $161(67.6)$ & $<0.0001^{\mathrm{a}}$ \\
\hline \multicolumn{6}{|l|}{ Indication for pacemaker } \\
\hline AVB & $603 / 1,205(50.0)$ & $570 / 1,145(49.8)$ & $9(42.9)$ & $24(61.5)$ & - \\
\hline AF brady & $76 / 1,205(6.3)$ & $75 / 1,145(6.6)$ & $0(0.0)$ & $1(2.6)$ & \\
\hline Others & $22 / 1,205(1.8)$ & $12 / 1,145(1.0)$ & $3(14.3)$ & 7 (17.9) & \\
\hline \multicolumn{6}{|l|}{ Indication for ICD } \\
\hline Secondary prevention & $340 / 655(51.9)$ & - & $273 / 429(63.6)$ & $67 / 226(29.6)$ & - \\
\hline Primary prevention & $315 / 655(48.1)$ & - & $156 / 429(36.4)$ & $159 / 226(70.4)$ & \\
\hline DCM & $169 / 1,786(9.5)$ & $7 / 1,132(0.6)$ & $39 / 429(9.1)$ & $123 / 225(54.7)$ & \\
\hline Brugada syndrome & $81 / 1,786(4.5)$ & $0 / 1,132(0.0)$ & $81 / 429(18.9)$ & $0 / 225(0.0)$ & \\
\hline $\mathrm{HCM}$ & $82 / 1,786(4.6)$ & $18 / 1,132(1.6)$ & $61 / 429(14.2)$ & $3 / 225(1.3)$ & \\
\hline Cardiac sarcoidosis & $62 / 1,786(3.5)$ & $11 / 1,132(1.0)$ & $27 / 429(6.3)$ & $24 / 225(10.7)$ & \\
\hline IVF & $39 / 1,786(2.2)$ & $0 / 1,132(0.0)$ & $39 / 429(9.1)$ & $0 / 225(0.0)$ & \\
\hline DHCM & $25 / 1,786(1.4)$ & $0 / 1,132(0.0)$ & $9 / 429(2.1)$ & $16 / 227(7.1)$ & \\
\hline ARVC & $17 / 1,786(1.0)$ & $0 / 1,132(0.0)$ & $15 / 429(3.5)$ & $2 / 227(0.9)$ & \\
\hline Others & $119 / 1,786(6.7)$ & $55 / 1,132(4.9)$ & $46 / 429(10.7)$ & $18 / 227(8.0)$ & \\
\hline Biotronik & 709 (38.3) & $586(50.2)$ & $100(22.6)$ & $23(9.7)$ & \\
\hline Abbot Medical & $182(9.8)$ & $142(12.2)$ & $15(3.4)$ & $25(10.5)$ & \\
\hline Boston Scientific & $98(5.3)$ & $19(1.6)$ & $60(13.5)$ & $19(8.0)$ & \\
\hline \multicolumn{6}{|c|}{$\begin{array}{l}\text { Values are the mean } \pm \text { standard deviation or number }(\%) \text { of } \\
\text { ap values were calculated using Pearson's chi-square test. } \\
\text { b p values were calculated using the Kruskal-Wallis test. }\end{array}$} \\
\hline
\end{tabular}

$5.0 \%$; CRT-D, 5.9\%) (Table 2). Battery depletion events included premature triggering of the elective replacement indicator in 7 Enrhythm (Medtronic) devices and one unexpected battery depletion in an ICD. With the exception of ICD therapy, there were no significant differences in the critical events among the three groups.

In the multivariate analysis to assess the risk of critical events, the independent risk factors for critical events were ICD (ICD vs. PM: odds ratio, 7.895; 95\% confidence interval, 3.360-18.553; p<0.0001), CRT-D (CRT-D vs. PM, odds ratio, 5.519; 95\% confidence interval, 2.249-13.548; $\mathrm{p}=$ 0.0002 ), and low ejection fraction (odds ratio, 0.986; $95 \%$ confidence interval, 0.973-0.998; $\mathrm{p}=0.0232$ ). Age, sex, baseline heart disease, and CIED manufacturer were not independent risk factors for critical events (Table 3).

In the multivariate analysis, the risk factors for critical events in the PM and CRT-D groups could not be analyzed due to the low number of critical events. In patients with ICDs, the independent risk factors for critical events included old age, low ejection fraction, Brugada syndrome, dilated phase hypertrophic cardiomyopathy, and arrhythmogenic right ventricular cardiomyopathy rather than ischemic heart disease (Table 4).

\section{Discussion}

\section{Main findings}

The main finding of this retrospective cohort study was that the critical event rate was very low, although abnormal events were identified in approximately two-thirds of all data transmissions. In addition, the critical event rate in PM 
Table 2. Transmission Data Characteristics by Devices.

\begin{tabular}{|c|c|c|c|c|c|}
\hline Variable & Overall & PM & ICD & CRT-D & $\mathrm{p}$ value ${ }^{\mathrm{b}}$ \\
\hline Number of transmission & 16,565 & 7,634 & 5,911 & 3,015 & - \\
\hline \multicolumn{6}{|l|}{ Abnormal event } \\
\hline Overall & $11,040(66.7)$ & $4,626(60.6)$ & $3,900(66.0)$ & $2,514(83.4)$ & $<0.0001$ \\
\hline ATA & $5,981(36.1)$ & $3,317(43.5)$ & $1,423(24.1)$ & $1,241(41.2)$ & $<0.0001$ \\
\hline VTA & $3,753(22.7)$ & $1,392(18.2)$ & $1,423(24.1)$ & $938(31.1)$ & $<0.0001$ \\
\hline Abnormal battery voltage event ${ }^{\mathrm{a}}$ & $334(2.0)$ & $55(0.7)$ & $180(3.0)$ & $99(3.3)$ & $<0.0001$ \\
\hline \multicolumn{6}{|l|}{ Critical event } \\
\hline Overall & $676(4.1)$ & $66(0.9)$ & $390(6.6)$ & $220(7.3)$ & $<0.0001$ \\
\hline Battery depletion & $19(0.1)$ & $7(0.1)$ & $8(0.1)$ & $4(0.1)$ & - \\
\hline ICD therapy & $476(2.9)$ & - & $298(5.0)$ & $178(5.9)$ & - \\
\hline Appropriate therapy & $374 / 465(80.4)$ & - & $239 / 292(81.8)$ & $135 / 173(78.0)$ & - \\
\hline Inappropriate therapy & $91 / 465(19.6)$ & - & $53 / 292(18.2)$ & $38 / 173(22.0)$ & - \\
\hline Lead impedance abnormality & $36(0.2)$ & $19(0.2)$ & $13(0.2)$ & $4(0.1)$ & - \\
\hline Non-physiologic noise & $86(0.5)$ & $24(0.3)$ & $42(0.7)$ & $20(0.7)$ & - \\
\hline Sensing failure & $11(0.1)$ & $6(0.1)$ & $3(0.1)$ & $2(0.1)$ & - \\
\hline Pacing failure & $6(0.0)$ & $6(0.1)$ & $0(0.0)$ & $0(0.0)$ & - \\
\hline Acute reduced atrial or ventricular sensing & $5(0.0)$ & $3(0.0)$ & $1(0.0)$ & $1(0.0)$ & - \\
\hline Acute increased atrial or ventricular pacing threshold & $31(0.2)$ & $6(0.1)$ & $17(0.3)$ & $8(0.3)$ & - \\
\hline Sustained VT out of therapy zone & $19(0.1)$ & - & $10(0.2)$ & $9(0.3)$ & - \\
\hline Others & $11(0.1)$ & $0(0.0)$ & $8(0.1)$ & $3(0.1)$ & - \\
\hline
\end{tabular}

Values are the number (\%) of patients. The sum of the percentages may not equal $100 \%$ because of rounding.

aSix missing data were excluded from the analysis.

${ }^{\mathrm{b}} \mathrm{p}$ values were calculated using a generalized estimating equation with a compound symmetry working correlation structure that accounted for associations between repeated measures within subjects.

PM: pacemaker, ICD: implantable cardioverter defibrillator, CRTD: cardiac resynchronization therapy defibrillator, ATA: atrial tachyarrhythmia, VTA: ventricular tachyarrhythmia

Table 3. Multivariate Analysis to Estimate the Correlations of Critical Events with Covariates.

\begin{tabular}{lcc}
\hline \multicolumn{1}{c}{ Variable (Definition of Odds Ratio) } & Odds Ratio [95\% CI] & p value \\
\hline Age & $1.008[0.997-1.020]$ & 0.1487 \\
Gender (Female/Male) & $0.705[0.490-1.015]$ & 0.0604 \\
Device (ICD/PM) & $7.895[3.360-18.553]$ & $<0.0001$ \\
Device (CRT-D/PM) & $5.519[2.249-13.548]$ & 0.0002 \\
Company (B/A) & $1.002[0.707-1.420]$ & 0.9905 \\
Company (C/A) & $1.161[0.694-1.943]$ & 0.5692 \\
Company (D/A) & $1.418[0.822-2.444]$ & 0.2090 \\
Baseline heart disease (IHD/None) & $0.490[0.201-1.197]$ & 0.1178 \\
Baseline heart disease (DCM/None) & $0.968[0.385-2.432]$ & 0.9448 \\
Baseline heart disease (Brugada syndrome/None) & $0.932[0.338-2.569]$ & 0.8920 \\
Baseline heart disease (HCM/None) & $0.693[0.240-1.999]$ & 0.4978 \\
Baseline heart disease (Cardiac sarcoidosis/None) & $0.884[0.336-2.324]$ & 0.8027 \\
Baseline heart disease (IVF/None) & $0.864[0.295-2.535]$ & 0.7903 \\
Baseline heart disease (DHCM/None) & $1.526[0.429-5.425]$ & 0.5140 \\
Baseline heart disease (ARVC/None) & $1.648[0.555-4.895]$ & 0.3682 \\
Baseline heart disease (Others/None) & $0.845[0.351-2.035]$ & 0.7074 \\
Atrial lead (With/Without) & $1.227[0.755-1.994]$ & 0.4078 \\
EF & $0.986[0.973-0.998]$ & 0.0232 \\
\hline
\end{tabular}

Company A, B, C and D indicated Medtronic, Biotronik, Abbot Medical and Boston Scientific, respectively.

CI: confidence interval, PM: pacemaker, ICD: implantable cardioverter defibrillator, CRT-D: cardiac resynchronization therapy defibrillator, IHD: ischemic heart disease, DCM: dilated cardiomyopathy, HCM: hypertrophic cardiomyopathy, DHCM: dilated phase hypertrophic cardiomyopathy, IVF: idiopathic ventricular fibrillation, ARVC: arrhythmogenic right ventricular cardiomyopathy, EF: ejection fraction 
Table 4. Multivariate Analysis to Estimate the Factors Correlated with Critical Events in Patients with ICDs.

\begin{tabular}{lcc}
\hline \multicolumn{1}{c}{ Variable (Definition of Odds Ratio) } & Odds Ratio [95\% CI] & $\mathrm{p}$ value \\
\hline Age & $1.017[1.003-1.031]$ & 0.0183 \\
Gender (Female/Male) & $0.690[0.395-1.204]$ & 0.1910 \\
Company (B/A) & $1.136[0.714-1.808]$ & 0.5898 \\
Company (C/A) & $1.855[0.596-5.772]$ & 0.2861 \\
Company (D/A) & $1.428[0.705-2.893]$ & 0.3231 \\
Baseline heart disease (DCM/IHD) & $1.073[0.534-2.160]$ & 0.8424 \\
Baseline heart disease (Brugada syndrome/IHD) & $2.323[1.099-4.912]$ & 0.0274 \\
Baseline heart disease (HCM/IHD) & $1.631[0.713-3.730]$ & 0.2462 \\
Baseline heart disease (Cardiac sarcoidosis/IHD) & $1.864[0.812-4.278]$ & 0.1418 \\
Baseline heart disease (IVF/IHD) & $2.254[0.951-5.342]$ & 0.0649 \\
Baseline heart disease (DHCM/IHD) & $5.579[1.441-21.604]$ & 0.0128 \\
Baseline heart disease (ARVC/IHD) & $4.125[1.772-9.604]$ & 0.0010 \\
Baseline heart disease (Others/IHD) & $2.519[1.187-5.347]$ & 0.0161 \\
EF & $0.978[0.963-0.994]$ & 0.0080 \\
Indication for ICD (Primary/Secondary) & $1.031[0.665-1.598]$ & 0.8918 \\
\hline Company A, B, C and D indicated Medtronic, Biotronik, Abbot Medical and Boston Scientific, re- \\
spectively. & \\
CI: confidence interval, PM: pacemaker, ICD: implantable cardioverter defibrillator, CRT-D: cardi- \\
ac resynchronization therapy defibrillator, IHD: ischemic heart disease, DCM: dilated cardiomyop- \\
athy, HCM: hypertrophic cardiomyopathy, DHCM: dilated phase hypertrophic cardiomyopathy, \\
IVF: idiopathic ventricular fibrillation, ARVC: arrhythmogenic right ventricular cardiomyopathy, \\
EF: ejection fraction
\end{tabular}

patients was significantly lower than that in ICD or CRT-D patients. The patients with a low ejection fraction were likely to have critical events. The expert consensus statement proposed in-hospital visits every 6-12 months for patients with PM. If the selection of patients for CIED follow-up could be limited to those who have experienced a critical event, the workload would decrease dramatically. This study is the first report to examine the factors, including devices made by various manufacturers and types of CIEDs, associated with high critical event rates in "real-world" CIED patients.

\section{Definitions of critical events}

The critical events are defined in the Methods. ATA events that required anticoagulation therapy and heart failure-related events were excluded because the strategy for intervention was not indicated, which led to a large difference in the intervention rate that was dependent on physicians. Recent CIEDs have many parameters that require precise tuning, such as AV delay, VV delay, refractory period, blanking period, pacing mode, rate response, automatic pacing threshold testing, and automatic sensitivity setting, which also showed a major difference in the intervention rate that was dependent on the physician. Although these data might have been related to the clinical intervention rate, this study only focused on critical events that definitely required intervention. Thus, the critical event rate in the present study might have been underestimated.

\section{The critical event rate in previous reports}

As reported by Facchin et al. (16), the critical event rate was very low in the PM population. They reported that $1,882(38 \%)$ patients had at least one clinically relevant event that required further investigation among a total of 4,965 remote transmissions; however, only 137 interventions (2.8\%) were required after further investigation by expert nurses and doctors. Their critical event rate was slightly higher than that in the present study because AF events were excluded from critical events. Ricci et al. (17) also reported that $133(6 \%)$ patients consulted a physician for further clinical evaluation, and $55(2 \%)$ required additional intervention to restore transmission interruption of the 2,249 analyses performed by nurses, which was similar to the present results. However, the types of CIEDs in the population, which included PM, ICD, and CRT-D patients, were not analyzed.

In the present study, patients with a low ejection fraction were likely to have critical events, which was in accordance with previous reports. As previously reported, patients with a low ejection fraction were likely to have appropriate ICD therapies (18-20), which was closely associated with critical events.

Several studies have analyzed the transmitted data of different types of CIEDs. Lazarus (21) reported that the mean numbers of events per patient per month reported by Home Monitoring to caregivers were 1.1, 0.7, and 2.1 (overall average, 0.6), for PM, ICD, and CRT-D recipients, respectively. In the ATHENS multicenter registry (22), the preva- 
lence of "visit with action" (corresponding to the present "critical event") in the PM, ICD, and CRT groups was $22.8 \%, 18.6 \%$, and $29.8 \%$, respectively. In these studies, the event rate was lowest in the ICD group, but the definition of critical event was different from that of the present study. The patient characteristics, patient population, and the underlying diseases of the enrolled patients could account for the differences between our study and these previous studies.

The present study showed that the critical event rate did not differ according to device manufacturer. This result could imply that the RM system would be effective in managing the follow-up of CIED patients, regardless of the device manufacturer. de Ruvo et al. (23) compared daily RM transmission systems (BHM) to periodic RM transmission systems (BSL, MCL, and AMM) and noted that daily transmission was associated with a higher cumulative rate of actionable events. In the present study, the proportion of ICD patients with BHM was relatively low, while that of PM patients with BHM was relatively high. In addition to the different definitions of critical events, this fact might have affected the present results..

In the ECOST trial (24), RM of ICD patients reduced costs, reducing the scheduled or additional ambulatory device evaluations per patient-year in an RM follow-up group in comparison to a conventional follow-up group. Although the present study did not show the cost-effectiveness of RM follow-up, the low critical event rate might lead to indirect cost savings.

In the ICD group, independent risk factors for critical events included Brugada syndrome, dilated phase hypertrophic cardiomyopathy, and arrhythmogenic right ventricular cardiomyopathy rather than ischemic heart disease. In patients with Brugada syndrome, it is reported that in addition to ICD therapy, adverse events, such as inappropriate ICD therapy, lead failure, or infection often occurred $(25,26)$. In patients with arrhythmogenic right ventricular cardiomyopathy, it is also reported that critical events associated with $\operatorname{ICD}$ are likely to occur $(27,28)$. However, no previous reports have compared the critical event rates among these groups.

\section{Limitations}

The present study was associated with some limitations. First, it was a multicenter, retrospective study that included a moderately sized study population. A prospective study with a larger study population could increase the reliability of these results. Second, only transmitted data and limited patient background data were analyzed in this study, whereas other data, such as comorbidities, the prognosis, and medications, were not evaluated, which have revealed other categories that should receive attention. Third, the frequency of transmissions differed among hospitals and manufacturers, which might have affected the critical event rate. However, the transmission interval was within the recommended values of between 1 and 4 months; thus, we con- sider that a reliable database was maintained. Fourth, in the present study, only data transmitted by RM were analyzed, real intervention events in the outpatient clinic were not. Thus, the real intervention rate may differ from the critical event rate of the present study. Lastly, the device programming and management was non-standardized, which might have also influenced the critical event rate. However, the data in this study shows "real-world" results.

\section{Conclusion}

Although abnormal events were observed in approximately two-thirds of all transmitted RM data, the critical event rate was $<1 \%$ in the PM group, which was lower in comparison to the ICD and CRT-D groups. A low ejection fraction was an independent predictor of critical events. If the selection of patients with CIEDs for follow-up could be limited to those with critical events, the workload would decrease dramatically.

\section{The authors state that they have no Conflict of Interest (COI).}

\section{Acknowledgement}

The authors thank Motomi Tachibana, MD, PhD, Kohei Miyaji, MD, PhD, Shigeki Hiramatsu, MD, PhD, Akira Ueoka, MD, Keisuke Okawa, MD, Etsuko Ikeda, MD, Takefumi Oka, $\mathrm{MD}, \mathrm{PhD}$, Kenji Kawamoto, MD, Tadashi Wada, MD, PhD, Shingo Hosogi, MD, PhD, Mika Nishimoto, MD, PhD, Masamichi Tanaka, MD, PhD, Chisato Suezawa, MD, PhD, Sho Tsushima, MD, Kenki Enko, MD, PhD, Yasufumi Kijima, MD, $\mathrm{PhD}$, Toru Miyoshi, MD, PhD, and Tohru Ohe, MD, PhD. The authors also thank Hirotaka Iguchi, Norihiro Nishiyama, Yuki Takenaka, Katsuaki Sakate, and Susumu Iwado for the medical engineering work and Yuko Kobayashi, Miyuki Fujiwara, and Masayo Ohmori for the secretarial work.

\section{References}

1. Varma N, Epstein AE, Irimpen A, Schweikert R, Love C; TRUST Investigators. Efficacy and safety of automatic remote monitoring for implantable cardioverter-defibrillator follow-up: the Lumos-T Safely Reduces Routine Office Device Follow-up (TRUST) trial. Circulation 122: 325-332, 2010.

2. Saxon LA, Hayes DL, Gilliam FR, et al. Long-term outcome after ICD and CRT implantation and influence of remote device followup: the ALTITUDE survival study. Circulation 122: 2359-2367, 2010.

3. Crossley GH, Boyle A, Vitense H, Chang Y, Mead RH. The CONNECT (Clinical Evaluation of Remote Notification to Reduce Time to Clinical Decision) trial: the value of wireless remote monitoring with automatic clinician alerts. J Am Coll Cardiol 57: 1181-1189, 2011.

4. Mabo P, Victor F, Bazin P, et al. A randomized trial of long-term remote monitoring of pacemaker recipients (the COMPAS trial). Eur Heart J 33: 1105-1111, 2012.

5. Wilkoff BL, Auricchio A, Brugada J, et al. HRS/EHRA expert consensus on the monitoring of cardiovascular implantable electronic devices (CIEDs): description of techniques, indications, personnel, frequency and ethical considerations. Heart Rhythm 5: 907-925, 2008 
6. Slotwiner D, Varma N, Akar JG, et al. HRS Expert Consensus Statement on remote interrogation and monitoring for cardiovascular implantable electronic devices. Heart Rhythm 12: e69-e100, 2015.

7. Dubner S, Auricchio A, Steinberg JS, et al. ISHNE/EHRA expert consensus on remote monitoring of cardiovascular implantable electronic devices (CIEDs). Europace 14: 278-293, 2012.

8. Masoudi FA, Ponirakis A, de Lemos JA, et al. Trends in U.S. Cardiovascular Care: 2016 Report From 4 ACC National Cardiovascular Data Registries. J Am Coll Cardiol 69: 1427-1450, 2017.

9. Qintar M, Zardkoohi O, Hammadah M, et al. The impact of changing antiseptic skin preparation agent used for cardiac implantable electronic device (CIED) procedures on the risk of infection. Pacing Clin Electrophysiol 38: 240-246, 2015.

10. Kanal E, Froelich J, Barkovich AJ, et al. Standardized MR terminology and reporting of implants and devices as recommended by the American College of Radiology Subcommittee on MR Safety. Radiology 274: 866-870, 2015.

11. Sanna T, Diener HC, Passman RS, et al. Cryptogenic stroke and underlying atrial fibrillation. N Engl J Med 370: 2478-2486, 2014.

12. Catanzariti D, Lunati $M$, Landolina $M$, et al. Monitoring intrathoracic impedance with an implantable defibrillator reduces hospitalizations in patients with heart failure. Pacing Clin Electrophysiol 32: 363-370, 2009.

13. Maines M, Catanzariti D, Cemin C, Vaccarini C, Vergara G. Usefulness of intrathoracic fluids accumulation monitoring with an implantable biventricular defibrillator in reducing hospitalizations in patients with heart failure: a case-control study. J Interv Card Electrophysiol 19: 201-207, 2007.

14. van Veldhuisen DJ, Braunschweig F, Conraads V, et al. Intrathoracic impedance monitoring, audible patient alerts, and outcome in patients with heart failure. Circulation 124: 1719-1726, 2011.

15. Bohm M, Drexler H, Oswald H, et al. Fluid status telemedicine alerts for heart failure: a randomized controlled trial. Eur Heart J 37: 3154-3163, 2016.

16. Facchin D, Baccillieri MS, Gasparini G, et al. Findings of an observational investigation of pure remote follow-up of pacemaker patients: is the in-clinic device check still needed? Int J Cardiol 220: 781-786, 2016.

17. Ricci RP, Morichelli L, Santini M. Home monitoring remote control of pacemaker and implantable cardioverter defibrillator patients in clinical practice: impact on medical management and health-care resource utilization. Europace 10: 164-170, 2008.

18. Brigadeau F, Kouakam C, Klug D, et al. Clinical predictors and prognostic significance of electrical storm in patients with implant- able cardioverter defibrillators. Eur Heart J 27: 700-707, 2006.

19. Streitner F, Kuschyk J, Veltmann C, et al. Predictors of electrical storm recurrences in patients with implantable cardioverterdefibrillators. Europace 13: 668-674, 2011.

20. Bergau L, Willems R, Sprenkeler DJ, et al. Differential multivariable risk prediction of appropriate shock versus competing mortality - a prospective cohort study to estimate benefits from ICD therapy. Int J Cardiol 272: 102-107, 2018.

21. Lazarus A. Remote, wireless, ambulatory monitoring of implantable pacemakers, cardioverter defibrillators, and cardiac resynchronization therapy systems: analysis of a worldwide database. Pacing Clin Electrophysiol 30(Suppl): S2-S12, 2007.

22. Mascioli G, Curnis A, Landolina M, et al. Actions elicited during scheduled and unscheduled in-hospital follow-up of cardiac devices: results of the ATHENS multicentre registry. Europace 13: 1766-1773, 2011.

23. de Ruvo E, Sciarra L, Martino AM, et al. A prospective comparison of remote monitoring systems in implantable cardiac defibrillators: potential effects of frequency of transmissions. J Interv Card Electrophysiol 45: 81-90, 2016.

24. Guedon-Moreau L, Lacroix D, Sadoul N, et al. A randomized study of remote follow-up of implantable cardioverter defibrillators: safety and efficacy report of the ECOST trial. Eur Heart J 34: 605-614, 2013.

25. Hernandez-Ojeda J, Arbelo E, Borras R, et al. Patients with Brugada syndrome and implanted cardioverter-defibrillators: long-term follow-up. J Am Coll Cardiol 70: 1991-2002, 2017.

26. Gonzalez Corcia MC, Sieira J, Pappaert G, et al. Implantable cardioverter-defibrillators in children and adolescents with Brugada syndrome. J Am Coll Cardiol 71: 148-157, 2018.

27. Orgeron GM, James CA, Te Riele A, et al. Implantable cardioverter-defibrillator therapy in arrhythmogenic right ventricular dysplasia/cardiomyopathy: predictors of appropriate therapy, outcomes, and complications. J Am Heart Assoc 6: e006242, 2017.

28. Al-Ghamdi B, Mallawi Y, Shafquat A, et al. Appropriate and inappropriate implantable cardioverter defibrillators therapies in arrhythmogenic right ventricular cardiomyopathy/dysplasia patients. Cardiol Res 9: 204-214, 2018.

The Internal Medicine is an Open Access journal distributed under the Creative Commons Attribution-NonCommercial-NoDerivatives 4.0 International License. To view the details of this license, please visit (https://creativecommons.org/licenses/ by-nc-nd/4.0/).

(C) 2019 The Japanese Society of Internal Medicine Intern Med 58: 2333-2340, 2019 